\title{
Aerodynamic shape optimization of aircraft engine nozzles based on Computer-Aided Design
}

\author{
Simon Bagy* and Mathieu Lallia ${ }^{\dagger}$ and Pascal Coat ${ }^{\ddagger}$ \\ Safran Aircraft Engines, F-77550 Moissy-Cramayel, France \\ Bijan Mohammadi ${ }^{\S}$ \\ Université de Montpellier, F-34090 Montpellier, France \\ Michaël Méheut ${ }^{\text {II }}$ \\ ONERA - The French Aerospace Lab, F-92190 Meudon, France
}

This study presents nozzle and cowl shape optimizations for turbofan separate-jet engines. The main objective of this work is to implement an optimization process including a detailed Computer-Aided Design (CAD) model and improve nozzles propulsive performance, taking into account the complete geometry of the aft-engine body. Integrating detailed CAD models in optimization workflows remains challenging, that is why an original approach is considered. This method uses expert knowledge to reduce dimensionality and enables to compute sensitivities with finite differences. The results illustrate the interest of this innovative industrial optimization approach for the design of turbofan nozzles.

\section{Nomenclature}

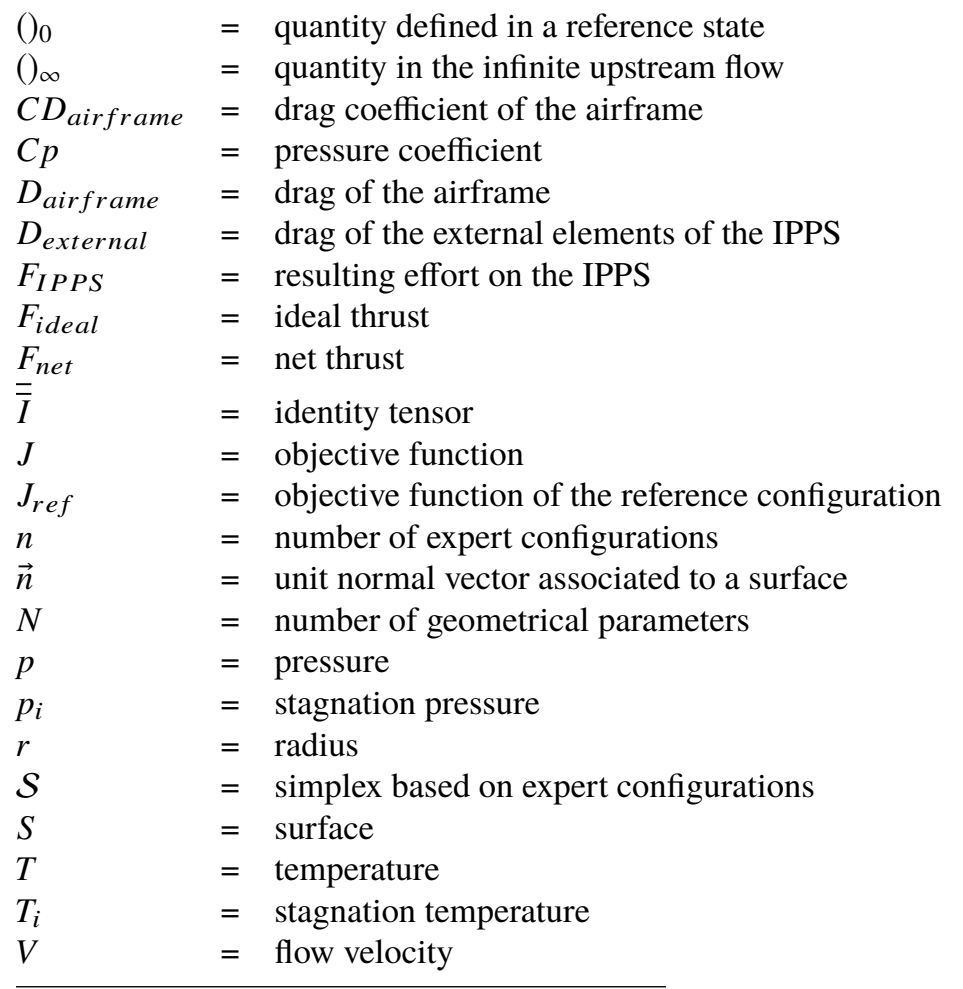

*PhD Student, simon.bagy@safrangroup.com

†Engineer, mathieu.lallia@safrangroup.com

Engineer, pascal.coat@ safrangroup.com

§Professor, Institut Montpelliérain Alexander Grothendieck, bijan.mohammadi@umontpellier.fr

IIDr. Research Engineer, Aerodynamics, Aeroelasticity, Acoustics Department, Civil Aircraft Unit, michael.meheut@onera.fr 


$\begin{array}{ll}X & =\text { design } \\ X_{i} & =\text { expert configuration } i \\ X_{\text {target }} & =\text { target configuration for inverse optimization } \\ x & =\text { axial coordinate } \\ \vec{x} & =\text { axial unit vector } \\ \alpha_{\text {geom }} & =\text { geometrical design parameters } \\ \lambda_{i} & =\text { convex combination coefficient associated to configuration } i \\ \Lambda & =\text { vector of the convex combination coefficients } \\ \rho & =\text { density } \\ \overline{\bar{\sigma}} & =\text { stress tensor } \\ \overline{\bar{\tau}} & =\text { viscous stress tensor }\end{array}$

\section{Introduction}

In order to reduce the environmental footprint of air transport, aircraft and engine manufacturers are developing fuel-efficient systems for future configurations. To tackle this objective, it becomes necessary to have powerful and reliable design methods. Concerning the design of aerodynamic shapes, optimization approaches bring significant improvement at a reasonable computational cost [1]. By taking advantage of recent advances in computational capabilities, these methods are now associated with viscous flow computations and applied on geometries of industrial complexity [2]. This leads to a growing interest to integrate these methods in industrial design phases of aerodynamic systems.

In particular, the nozzles are located on the rear part of the engine and play a significant role in all phases of flight for operability and global engine performance. The rear-body elements, comprising nozzles and cowls, are commonly based on axisymmetrical shapes. However, several structural elements, such as the pylon or the bifurcations located in the fan nozzle, alter the symmetry of the nozzle flows. Furthermore, when the engines are installed under the wing, the nozzles operate in a highly-tridimensional aerodynamic environment. Consequently, taking the non-symmetrical character of the flow into account in the design process is an opportunity to further increase nozzles performance. In this context, optimization methods are expected to be efficient design approaches to adapt nozzle shapes to their complex aerodynamic environment.

If shape optimization in computational aerodynamics has been mostly dedicated to wing design [3-6], several studies have also applied these methods to engine nacelles (including inlet, external cowls and nozzles). In the 90's, Bell et al. [7] used an inverse optimization technique based on pressure distributions for the design of 2D and 3D nacelles with Euler flow computations. Later, Lambert [8] implemented Euler flow and adjoint solvers, to perform gradient-based shape optimizations of engine nacelles. This thesis work focused on reducing the drag of the inlet and external shapes of the nacelle. More recently, optimizations have been carried out by Toubin et al. [9] on a three dimensional nacelle geometry. Several optimization algorithms, gradient-free and gradient-based with adjoint, have been applied using Reynolds Averaged Navier-Stokes (RANS) computations of the flow in cruise and crosswind conditions. In 2018, Goulos et al. [10] performed optimizations on a complex two-dimensional after-body geometry, using a strategy based on response surface modeling and genetic algorithms, as well as RANS flow simulations. Besides, Gray et al. [11, 12] replaced usual aerodynamic simulations by coupled aeropropulsive simulations in their optimization process. This enables to take into account the mutual effects between the aerodynamics and the thermodynamical operating point of the engine.

These studies show that aerodynamic shape optimization has evolved over the years and that it is now possible to perform high-fidelity flow computations or coupled aeropropulsive simulations in an optimization framework. However, despite their growing complexity, the geometries considered remain unsufficient for several reasons. First, single-flow nozzles do not represent the complexity of separate-exhaust industrial configurations. Then, axisymmetrical geometries do not enable to take three-dimensional elements into account. Moreover, these optimization workflows are mostly based on analytical shape parameterization or mesh deformation. Although these methods have significant advantages for optimization processes, in particular as they provide easy access to sensitivities, they do not comply with industrial design tools and parameters.

In this paper, CAD-based aerodynamic shape optimizations of dual-separate-flow turbofan nozzles are presented.

At first, a parameterized CAD model used to design complex nacelle configurations is described. An optimization method is then introduced, that enables the use of gradient-based optimization with such CAD tool. Next, a first 
assessment of the optimization approach is made on a simple inverse optimization problem. Then, the parameterized CAD model is used with the developed optimization method to improve the performance of an aircraft engine.

\section{CAD-based nacelle geometry and dedicated optimization approach}

\section{A. Detailed tridimensional geometry of an engine nacelle}

Prior to the optimization work, a CAD model has been developed using CATIA V5 (property of Dassault Systèmes [13]). This model, depicted on Fig. 1(a), is a geometric representation of the Integrated PowerPlant System (IPPS) of a three-dimensional turbofan engine with dual-separate-flow nozzles. It comprises the inlet, the outer nacelle, the core (primary) and fan (secondary) ducts and nozzles, as well as the pylon and the lower bifurcation located in the secondary flow. Three planes are defined at the interfaces with engine components. They are located upstream of the fan, downstream of the turbine in the core-flow, and downstream of the outlet guide-vane in the secondary flow. These planes are used to define boundary conditions for the flow solver.

The geometry of this model is generated from a table of parameters $\alpha_{\text {geom }}$, which are geometrical parameters based on expert-knowledge and engineering know-how. They have been defined to enable the generation of tridimensional nozzles and cowl shapes. For example, Fig. 1(b) illustrates the ability of the model to create highly deformed rear-body geometries. Consequently, a significant number of parameters is necessary and the model involves approximately 300 variables.

Besides, the nozzles throat areas are fixed by the engine thermodynamic cycle and must be respected during nozzle design. Therefore, an intern loop in the model enables to adapt the nozzle geometry, in order to respect these constraints despite non-axisymmetrical shapes.

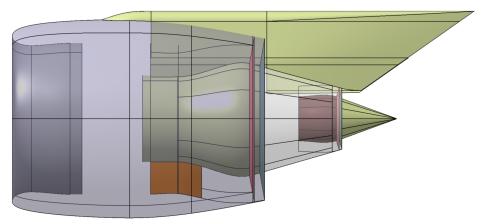

(a)

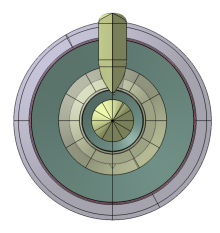

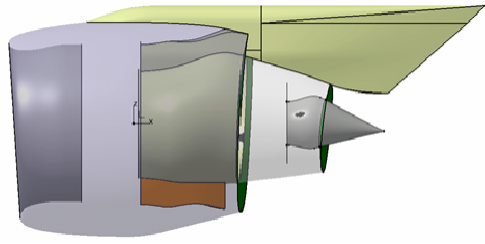

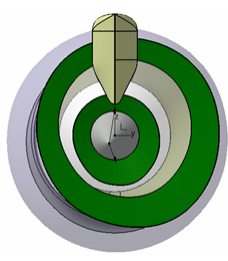

(b)

Fig. 1 High-fidelity CAD model of the nacelle in standard (a) and highly deformed (b) cases

\section{B. Challenges of CAD-based optimization}

Shape optimization on industrial CAD-based geometries remains challenging for several reasons. First, the high number of parameters involved represent a significant barrier for many optimization methods. Gradient-free methods have a rapidly increasing cost with respect to the dimension of the problem. For an application with hundreds of variables, the computational cost of these algorithms becomes prohibitive. In gradient-based optimizations, adjoint methods are usually used to tackle the high-dimensionality and compute derivatives. However, in this study, the industrial CAD software is used without access to the source code or the derivatives. Consequently, in order to use gradient-based optimization, an approach is proposed to reduce the dimension of the design space and compute derivatives with finite differences.

\section{Method of convex combination based on expert knowledge}

In the presented case, the design tool of the aircraft engine nacelle is an industrial CAD modeler and the number of parameters $\alpha_{\text {geom }}$ associated to the model is $N \sim 10^{2}$. Industrial know-how and expert knowledge are used to define $n$ reference shapes $X_{\{i=1, \ldots, n\}}$ in the admissible domain, with $n<<N$ (usually, $n$ is comprised between 3 and 10). These shapes are referred as "expert configurations".

Then, a convex set is defined as a simplex $\mathcal{S}$ based on these configurations:

$$
\mathcal{S}=\left\{\sum_{i=1}^{n} \lambda_{i} X_{i} \mid \sum_{i=1}^{n} \lambda_{i}=1 \text { and } \lambda_{i} \geq 0 \quad \forall i\right\}
$$


where $\lambda_{i} \in[0,1]$ are the barycentric coordinates.

The idea of this method, introduced for the first time in Bagy et al.[14], is to perform optimization over the convex set, instead of the complete design space. Thus, the dimension of the problem is reduced to $n$ and the global design variables have been replaced with $\Lambda=\left\{\lambda_{1}, \ldots, \lambda_{n}\right\} \in[0,1]^{n}$.

This approach is particularly interesting for preliminary design: defining the design space as a combination of expert configurations integrates expert knowledge into the optimization workflow and strongly reduces the risk of getting "industrially" unfeasible designs. Moreover, this method proposes an innovative way to explore a high dimensional design space. The complexity of the shape, that needs a high number of parameters, is not reduced; only the way to drive the exploration changes. Because the new search space is low-dimensional, functional sensitivities can be evaluated using finite differences. This also reduces the computational cost of gradient-free techniques and makes them affordable. Finally, the convex set exploration can be considered as the first step of a larger optimization framework. The optimal shape obtained on the convex set can be used as a starting point for a second optimization over the entire search space, with a method dedicated to high-dimensionality.

The drawback of this method is that the shapes generated on the convex set are limited by the expert configurations, but further improvements can be imagined to tackle this issue.

\section{Application of the convex combination on a simple case}

In order to demonstrate the abilities and the relevance of the convex combination approach, a test case representing a simple nozzle problem is defined.

Let $r$ be an analytic function defined on [0,1] that represents the radius of an $x$-axisymmetric nozzle wall (see Fig. 2(a) $X$ is the curve associated to $r$. An inviscid fluid flows through this nozzle. Only the mean flow values in $x$ sections are considered, so that the problem becomes mono-dimensional and only depends on $x$. Assuming that the flow is steady, incompressible, irrotational and isentropic, Bernoulli's equation applies [15]. Introducing $(\rho, p, V)$ as the flow density, static pressure and axial velocity at a given $x$, this yields:

$$
\forall x \in[0,1], \frac{V(x)^{2}}{2}+\frac{p(x)}{\rho}=\frac{V_{0}^{2}}{2}+\frac{p_{0}}{\rho}
$$

where the 0 -state is a reference upstream state of the nozzle flow. Thanks to the mass-flow conservation, $V$ can be related to the nozzle section $S$, that is expressed with respect to $r$ :

$$
\forall x \in[0,1], V(x)=\frac{V_{0} \cdot S_{0}}{\pi \cdot r(x)^{2}}
$$

Therefore, when $V$ is replaced in Eq. 2, the pressure can be computed on every point of the wall with:

$$
\forall x \in[0,1], p(x)=p_{0}+\frac{\rho \cdot V_{0}^{2}}{2}\left(1-\left(\frac{S_{0}}{\pi \cdot r(x)^{2}}\right)^{2}\right)
$$

This first application is an inverse optimization problem. An arbitrary analytical function $X_{\text {target }}$ is chosen as the optimal wall design and the process aims at retrieving this shape, based on the associated pressure distributions in the nozzle. The coordinate $x$ is discretized on the interval $[0,1]$ to create a design vector $r_{i}$ of dimension $N=300$ :

$$
\forall i \in \llbracket 1, \ldots, N \rrbracket, x_{i}=\frac{i}{N} \text { and } r_{i}=r\left(x_{i}\right)
$$

Then, the cost function to be minimized is defined as:

$$
J=\sum_{i=0}^{N}\left(p\left(x_{i}\right)-p_{\text {target }}\left(x_{i}\right)\right)^{2}
$$

Four nozzle geometries denoted $\left\{X_{1}, X_{2}, X_{3}, X_{4}\right\}$ are considered as 'expert configurations'. These shapes are displayed on Fig. 2(a) Three of these shapes have a throat, at different locations and with different sections ; one has a constant section.

A simple gradient-based descent algorithm is implemented and used for the descents presented in this section. The gradient is computed at each iteration by finite differences and an elementary step size adaptation is used. The descent algorithm is stopped after 500 iterations. 
Two test cases are considered, which differ on their target shapes. At first, the target shape $X_{\text {target }}$ is defined as a combination of the expert configurations, in order to assess the capability of this method to retrieve a shape inside the convex space. The convergence of the descent algorithm, diplayed on Fig. 2(b), shows that it converges towards the optimum in approximately 300 gradient iterations. Moreover, the shapes observed on Fig. 2(a) confirm that the descent method is capable to find the optimal design. On this figure, the slight discrepancy between the optimal shape and the target is due to the low gradient values at the end of the search process. The descent slows and the limited number of iterations does not permit to have a complete match of the curves.
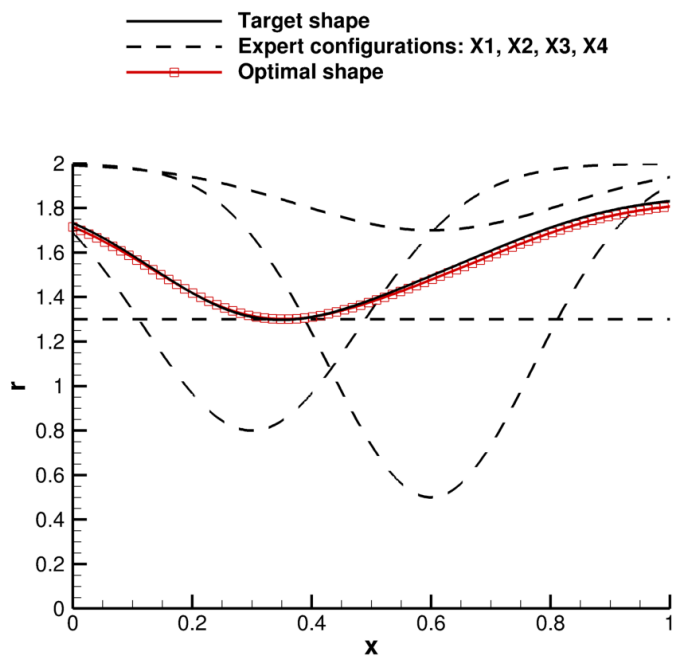

(a) Expert configurations, target and optimal nozzle shapes
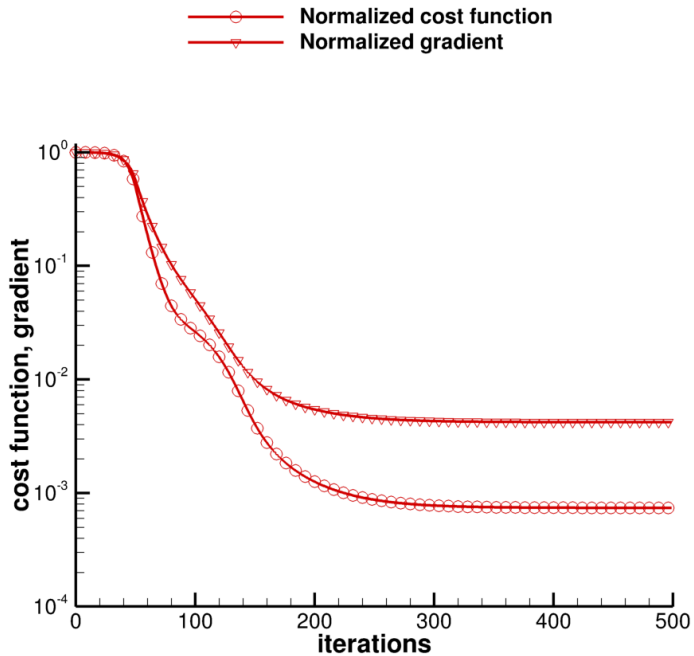

(b) Evolution of the normalized values of the cost function $J$ and its gradient $g$

Fig. 2 Gradient-based optimization on the space defined with convex combination, for a target shape belonging to the convex set

Then, the target shape is defined by a random analytical function that does not belong to the convex set. The descent on the reduced search space is compared to the descent in the full design space on this case. The optimal shapes and the convergence rates of the algorithm can be observed on Fig 3(a) and 3(b) respectively. It appears on Fig. 3(a) that the optimal shape on the full design space approaches more precisely the target shape than the shape defined as a convex combination of expert configurations. This result confirms that proposed method of search space reduction can limit the optimization search. However, despite the disparity of the expert configurations, a good convergence toward the solution can also be observed with the convex combination method. Moreover, the optimal combination is obtained in approximately 60 gradient iterations, compared to the 100 needed on the full design space. When considering the number of evaluations until convergence, the convex combination method appears to reduce drastically the cost of the process, totalizing 240 evaluations instead of 30,000. This is mainly due to the cost of gradient computations, needing $n=4$ evaluations on the reduced space instead of $N=300$ on the complete design space.

In conclusion, the proposed convex combination method has proven its ability of dealing with highly dimensional design spaces on a simple inverse optimization problem. This approach reduces significantly the cost of gradient computations for descent algorithms. Moreover, although the optimal shape obtained on the reduced space may not be as optimal as the result of the search on the full design space, it gives a good approximation of the optimum. Consequently, this method appears as an efficient way to approach the optimum for industrial CAD-based design problems. In the following, this method is applied to an industrial aerodynamic shape optimization problem. 


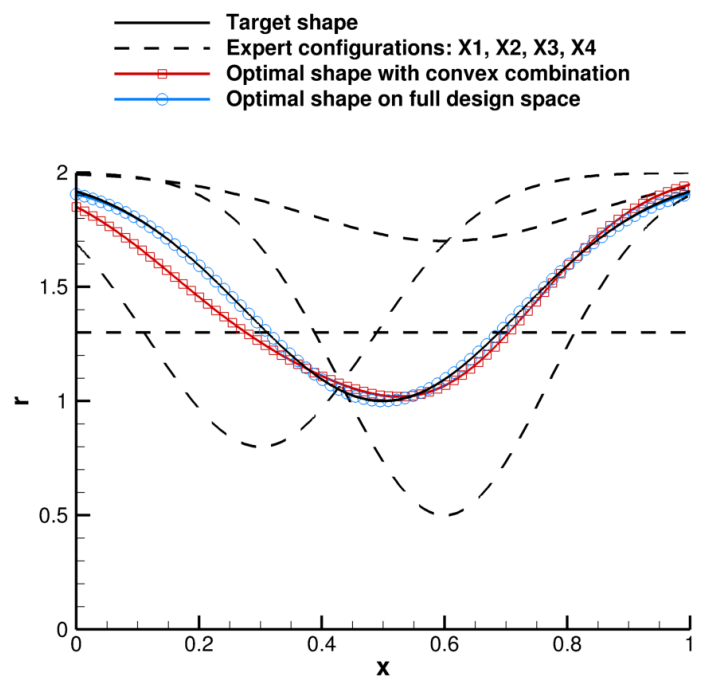

(a) Expert configurations, target and optimal nozzle shapes

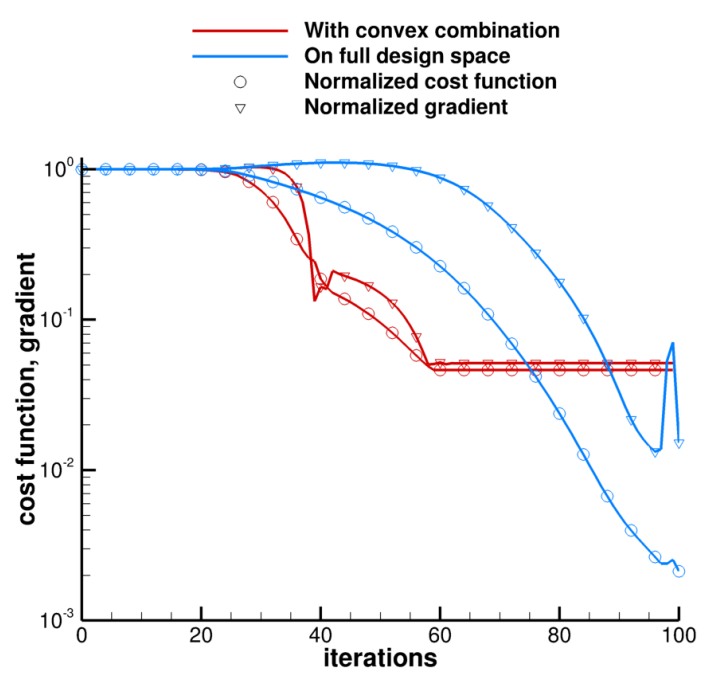

(b) Evolution of the normalized values of the cost function $J$ and its gradient $g$

Fig. 3 Comparison of gradient-based optimizations on the full design space and on the space defined with convex combination, for a target shape outside the convex set

\section{Industrial optimization problem for nozzle shapes}

\section{A. Case setup and aircraft/engine configuration}

This study proposes to perform shape optimization on the nozzles and the rear-body cowls of a turbofan engine. In order to be representative of an industrial design process, a target aircraft configuration is defined. The NOVA civil aircraft concepts designed at ONERA [16] are considered. Among the different NOVA concepts available, the configuration with underwing-mounted engines is considered as test case for this study. This aircraft is powered by Ultra-High By-Pass Ratio (UHBR) engines. An adapted engine configuration is proposed, based on the aircraft characteristics. Its properties are presented in Table 1

\section{Table 1 Main characteristics of the turbofan engine designed for the NOVA aircraft}

\begin{tabular}{l|c} 
Fan diameter & $85 \mathrm{in}$ \\
Fan Pressure Ratio & 1.3 \\
By-Pass Ratio & 16 \\
Sea Level Static Thrust & $23,800 \mathrm{lbf}$ \\
Cruise Mach number & 0.82 \\
Cruise altitude & $37,000 \mathrm{ft}$
\end{tabular}

However, the aircraft geometry is not included in the scope of the present work. Only the resulting drag effort on the aircraft without IPPS (sometimes called "glider") is taken into account to compute the thermodynamical operating point of the engine.

\section{B. Operating conditions of the engine}

In this work, the simulations of the IPPS are performed in cruise flight conditions, at an altitude of $37,000 \mathrm{ft}$, a Mach number of 0.82 and an angle of attack of $0^{\circ}$. The operating conditions of the engine can only be determined through an iterative process. The engine propulsive cycle gives flow conditions for the aerodynamic computations and the aerodynamic performance allows in turn to adjust the operating point of the cycle. 
Consequently, a thermodynamic model of the engine is developed using the software PROOSIS (property of Empresarios Agrupados[17]). This model enables to compute the values to be imposed in the boundary conditions of the Computational Fluid Dynamics (CFD) simulations, from aerodynamic post-treated quantities.

In cruise, at constant speed, a mechanical equilibrium can be established on the aircraft as:

$$
F_{I P P S}+D_{\text {airframe }}=0
$$

where $F_{I P P S}$ is the resulting effort on the complete powerplant, and $D_{\text {airframe }}$ is the drag of the airframe. The non-dimensional drag coefficient associated to the airframe in cruise flight is indicated by Wiart et al. [16] as $C D_{\text {airframe }}=266.2 \cdot 10^{-4}$.

Then, a nacelle shape (displayed on Fig. 7) is designed for this engine configuration. This shape is considered as "reference" for the rest of this study. Preliminary computations are performed on this geometry and a first guess of the operating conditions is made. Then, by iterating "manually", the operating conditions of the engine are adjusted until the mechanical equilibrium is verified. The resulting set of operating conditions is presented in Table 2

Table 2 Operating conditions of the reference engine in cruise flight

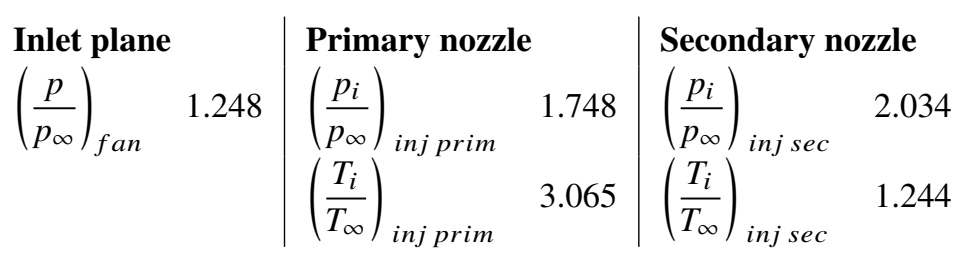

\section{Formulation of the optimization problem}

This work aims at improving the efficiency of the IPPS, comprising the engine, its nacelle and the pylon. In cruise conditions, this objective can be considered as lowering the fuel consumption while delivering thrust for the propulsion of the aircraft. As a first optimization case, no coupling is considered between aerodynamic computations and the thermodynamic propulsive model. Consequently, an adapted formulation of the optimization problem is used. It appears that improving engine efficiency is equivalent to maximizing thrust for given engine operating conditions.

The resulting effort on the powerplant, $F_{I P P S}$, is evaluated from aerodynamic computations. This effort is obtained by summing the net thrust delivered by the engine, $F_{n e t}$, and a drag term $D_{\text {external }}$. The net thrust is computed by integrating the efforts on the exhaust planes of the engine, on the rear cowls and the pylon surface licked by the engine flow, and substracting the effort in a plane located at the infinite upstream (see Fig 4):

$$
F_{n e t}=\int_{S_{\text {exhaust }}}\left[\rho \vec{V}(\vec{V} \cdot \vec{n})+\left(p-p_{\infty}\right) \vec{n}\right] \cdot \vec{x} d S+\int_{S_{\text {cowls }}}[\overline{\bar{\sigma}} \cdot \vec{n}] \cdot \vec{x} d S+\int_{S_{\text {pylon licked }}}[\overline{\bar{\sigma}} \cdot \vec{n}] \cdot \vec{x} d S-\int_{S_{\infty}}[\rho \vec{V}(\vec{V} \cdot \vec{n})] \cdot \vec{x} d S
$$

where $\overline{\bar{\sigma}}$ is the sum of the pressure and viscous stresses $\overline{\bar{\sigma}}=-p \overline{\bar{I}}+\overline{\bar{\tau}}$. Then, the external drag is defined as the integrated efforts on the external nacelle cowl and the pylon that is not licked by the engine flow:

$$
D_{\text {external }}=\int_{S_{\text {external }} \cup S_{\text {pylon external }}}[\overline{\bar{\sigma}} \cdot \vec{n}] \cdot \vec{x} d S
$$

In parallel, an ideal force is defined, based on the flow quantities injected in the nozzle planes. This term is computed by supposing that the flow is expanded isentropically until the infinite upstream pressure $p_{\infty}$ and is denoted $F_{\text {ideal }}$. Moreover, it takes into account the mass flow computed in the injection planes of the nozzle. Finally, the objective function $J$ to maximize is defined as:

$$
J=\frac{F_{I P P S}}{F_{\text {ideal }}}
$$

Thanks to the $F_{\text {ideal }}$ term, this objective function takes into account the intrinsic dependency of thrust on the mass flow rate. In addition to the area constraint in the geometrical model presented in III.A, this ensures that the efficiency is improved at a given mass flow rate and enables to avoid the definition of an optimization constraint on this quantity. 


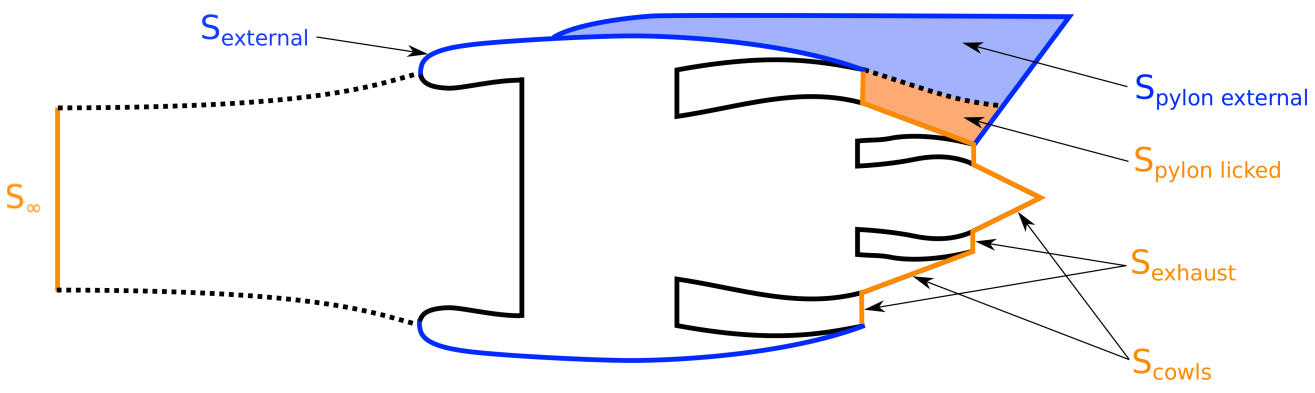

Fig. 4 Schematized view of the integration surfaces contributing to the resulting effort on the IPPS

\section{Optimization workflow and tools}

An optimization workflow that integrates industrial design tools is implemented for this study and displayed on Fig. 5. This process includes the previously introduced geometrical model, a mesh generation process based on CAD geometry and aerodynamic computations of the flow.

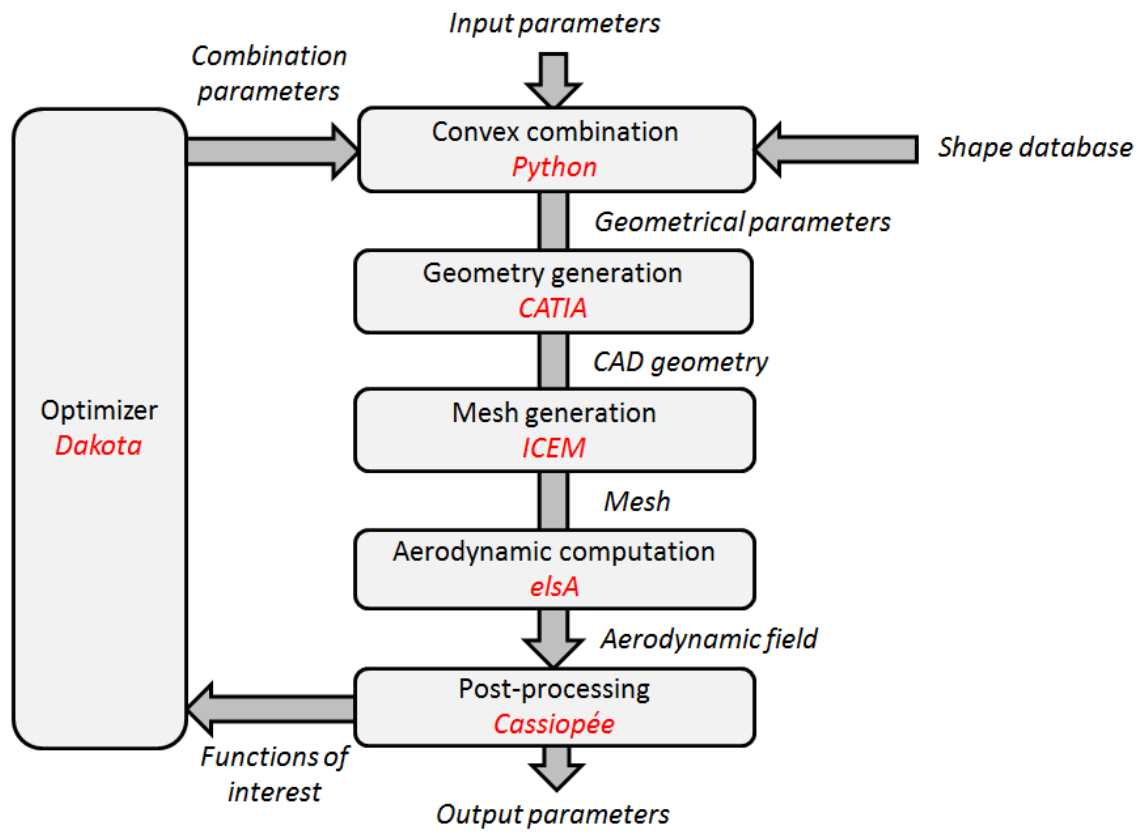

Fig. 5 Optimization workflow based on industrial tools

\section{Optimizer and algorithm}

This work consists in implementing the convex combination approach in an industrial optimization workflow. A dedicated step of the workflow generates the set of CAD parameters $\alpha_{\text {geom }}$ from the expert configuration parameters and the design variables $\lambda_{i=1 . . n}$. Still, as design variables are managed by the optimizer, a "convex" constraint is defined on the sum of the design variables:

$$
\sum_{i=1}^{n} \lambda_{i}=1
$$

This constraint has to be respected in order to be in the convex space $\mathcal{S}$. Consequently, the optimization problem considered is constrained.

At this point, it should be precised that although the convex combination method enables both gradient-free and gradient-based optimization processes, the choice is made to use gradient-based descent methods. Therefore, 
optimizations are performed with Dakota[18], using DOT's modified method of feasible descent [19] to explore the constrained design space.

\section{Mesh generation}

The CAD model presented in III.A generates a set of curves and points, that is loaded in the meshing software ICEM CFD (property of ANSYS [20]). A meshing process is defined following usual industrial procedures and is recorded as a macro. After a change in the design parameters, the CAD model is updated and the macro is replayed to re-create the 3D mesh.

The resulting mesh is a structured grid made of $15 \cdot 10^{6}$ cells. The near-wall region is refined for viscous computations, as well as the jet wake. Due to its number of cells, this grid is considered as coarse for final industrial applications. However, preliminary computations have shown that it enables relatively fast grid generation and flow computation, while being sufficiently accurate to evaluate nacelle aerodynamics with the precision needed for optimization.

The surface mesh of the nacelle is depicted on Fig. 6

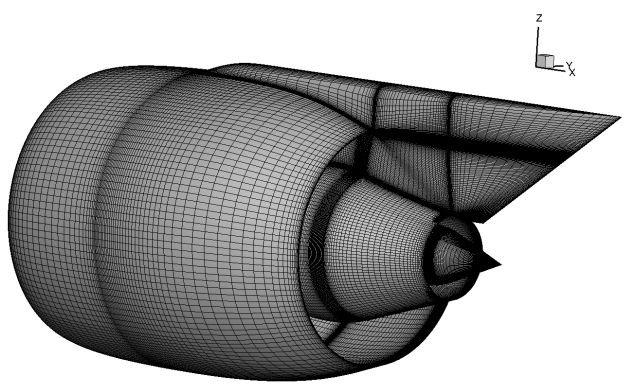

Fig. 6 Overview of the surface grid of the nacelle

\section{Aerodynamic computation}

The flow around the nacelle is computed using the elsA solver[21] (property of Airbus-Safran-ONERA). The numerical settings for CFD simulations are presented in Table 3 In addition, multigrid methods with one level of coarse grid are used to accelerate the convergence rate.

Table 3 Numerical parameters for flow computation

\begin{tabular}{l|l} 
Flow equations & RANS \\
Turbulence model & Spalart-Allmaras \\
Spatial scheme & Jameson \\
Pseudo-time stepping scheme & Backward Euler and LUSSOR implicit phase scalar
\end{tabular}

A post-processing based on Cassiopée[22] computes the quantities of interest from the aerodynamic field.

\section{E. Robustness of the workflow}

The first optimizations launched with the described workflow here have pointed out some issues in the process that prevented the good behaviour of the process. The main reasons for the errors were that:

- The CAD model did not manage to generate the geometries associated to some set of parameters.

- Even if the CAD model was acceptable, the meshing process generated some negative volume cells. In particular, these errors mostly occurred when the set of design variables required by the optimizer exceeded the value imposed to the convex constraint: $\sum_{i=0}^{n} \lambda_{i}=1$. Unfortunately, these steps can be necessary for the descent algorithm.

In order to manage these robustness issues, three main actions have been taken. First, the robustness of the CAD model and the associated mesh generation has been significantly improved. By generating a great number of shapes, several recurring errors of the CAD and the meshing have been identified. Corrective measures have been implemented in both the CAD model and the mesh replay. These adaptations enable a strong reduction of the number of errors. 
Then, the design variables associated to the inlet and the upstream external cowls have been fixed at a constant value. These design parameters are out of the scope of the present work, because they do not influence the rear-body and nozzles geometry. However, they can be altered if the convex constraint is not respected (i.e. $\sum_{i=0}^{n} \lambda_{i} \neq 1$ ). This appears as the source of several errors that happened on the CAD model. Consequently, considering them as non-modifiable in the workflow increases the robustness of the process.

Finally, it appears that despite the previous improvements, the optimization process sometimes requires sets of parameters that highly exceed the capabilities of any CAD model. This happens in particular when the optimizer moves away from the convex and $\left|\sum_{i=0}^{n} \lambda_{i}-1\right|>>0$. In order to adress this issue, an error management is implemented in the process. When the design variables lead to a degenerate geometry, a dummy value is set as objective function and the process continues.

To conclude, the adjustments made on the available CAD parameters, the geometrical model, and the meshing process increase the robustness of the shape generation and reduce the probability of getting degenerate geometries. In addition, an error managemenent is implemented to avoid a crash of the process when the optimizer exceeds the value of the convex constraint. Together, they enable the good operation of the optimization workflow.

\section{Optimized tubofan nozzle shapes}

The workflow based on CAD-generated nozzle shapes and aerodynamic flow computations is now used with the convex combination approach to perform optimization.

\section{A. Shape database for convex combination}

This work aims at assessing the convex combination approach on a first CAD-based workflow. Therefore, the choice has been made to choose only 3 expert configurations and to focus on the secondary nozzle shape. All shapes are based on a reference nacelle shape, displayed on Fig.7(a) In particular, the parameters related to the inlet and the external cowl of the engine remain unchanged during the optimization process. Only the shape parameters of the fan nozzle are altered and the ones defining the primary nozzle are common to all shapes.

The first shape in the database, $X_{1}$ (Fig. [7(b)], comprises a vertically ovalized nozzle. This shape introduces a non-axisymmetrical shape, that could benefit to the three-dimensional geometry. The second one, $X_{2}$, has an extended secondary flow nozzle compared to the reference (Fig. 7(c) ). On the contrary, the nozzle is shortened on the third geometry, $X_{3}$ (Fig. $7(\mathrm{~d})$ ). The two latter are based on axisymmetrical cowl geometries, on the contrary of the first one.

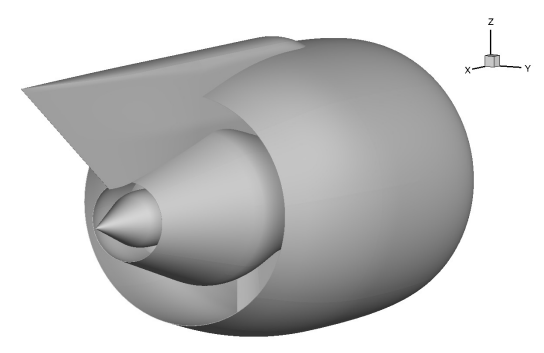

(a) Reference

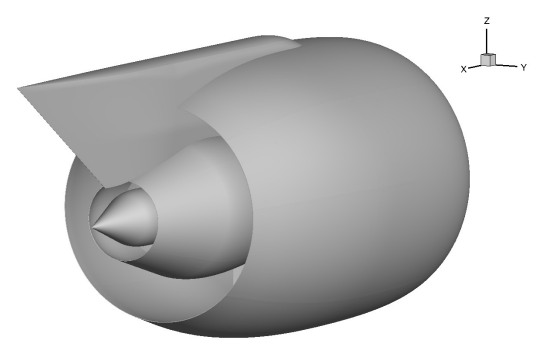

(c) Extended secondary nozzle $\left(X_{2}\right)$

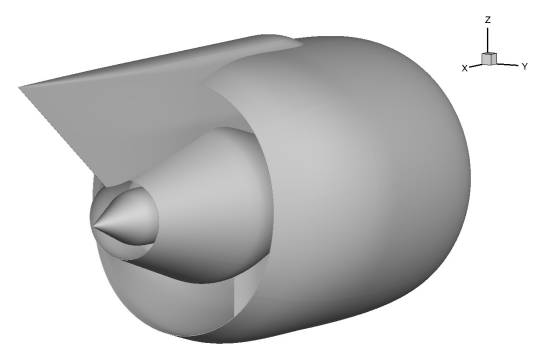

(b) Vertically ovalized secondary nozzle $\left(X_{1}\right)$

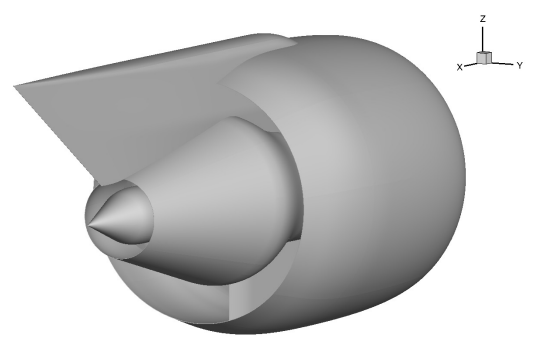

(d) Shortened secondary nozzle $\left(X_{3}\right)$

Fig. 7 Reference rear-body shape and "expert" configurations in the database for combination 


\section{B. Optimized nozzle shape}

The optimization process is launched from the barycenter of the convex space, at $\Lambda=(0.3333,0.3333,0.3333)$. After 2 gradient computations and a total of 41 evaluations, the descent algorithm converges. The resulting optimal shape enables an improvement of $0.9 \%$ on the objective function, with respect to the value of the reference nozzle shape (see comparison in Table 4 .

\section{Table 4 Performance comparison of the nozzle shapes}

\begin{tabular}{lc}
\hline Configuration & Objective function $J / J_{\text {ref }}$ \\
\hline Reference & 1.000 \\
Vertically ovalized secondary nozzle $\left(X_{1}\right)$ & 0.918 \\
Extended secondary nozzle $\left(X_{2}\right)$ & 1.004 \\
Shortened secondary nozzle $\left(X_{3}\right)$ & 0.963 \\
Optimal nozzle & 1.009 \\
\hline
\end{tabular}

The coefficients for optimal combination are $\Lambda=(0.000,0.757,0.243)$. Their values give valuable information to the designer on the configurations of the database. The shape $X_{1}$, with the ovalized nozzle, has completely been abandoned to the profit of the shapes that modified the nozzle length. Moreover, the fact that $\lambda_{2}>\lambda_{3}$ indicates a preference for the extended secondary nozzle.

Then, the nozzles shapes can be compared in a plane $z=0$, as displayed on Fig. 8 On this figure, it appears that the optimal shape has an extended secondary nozzle in comparison with the reference. This is in good agreement with the interpretation of the combination coefficients. Moreover, due to the design of the "expert" configurations in the database, this choice of coefficients also has an influence on the inner shape of the nozzle. The secondary duct appears "smoother" than on the shortened and the reference shapes.

$\begin{array}{ll}\text { Reference } & \text { Ovalized secondary nozzle } \\ \text { Optimal } & \text { Extended secondary nozzle } \\ & \text { Shortened secondary nozzle }\end{array}$
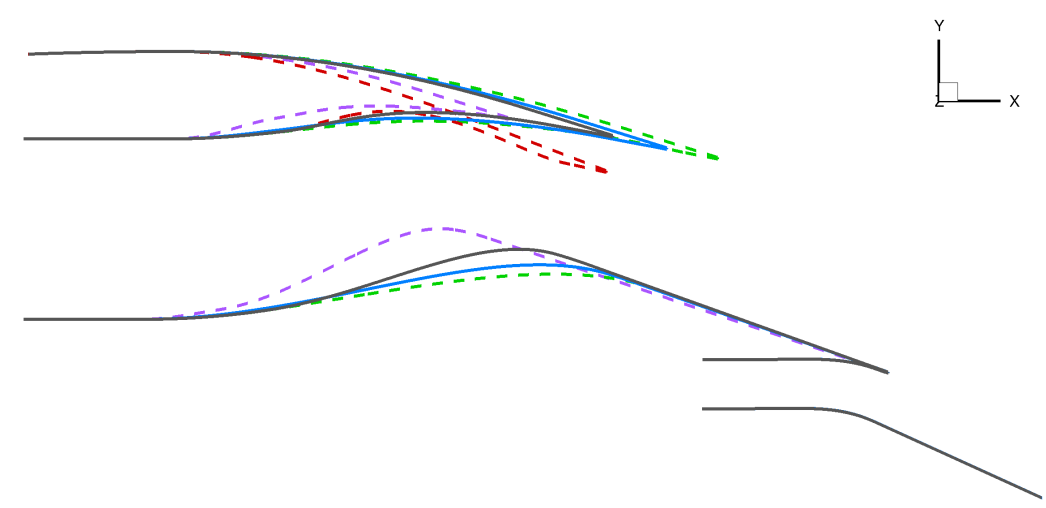

Fig. 8 Comparison of the nozzle geometries, for a slice in the $z=0$ plane

Next, a look at the Mach fields in the nozzles (Fig. 9) enables to have a physical understanding of the optimization result. The plot shows that the maximum Mach number in the optimal nozzle is lower than in the reference shape. This observation suggests that the Mach evolution along the secondary duct plays a role in the improvement of the objective function. By reducing the flow velocity, the optimizer also reduces the friction efforts on the nozzle wall, and increases the efficiency of the engine. At this point, a detailed breakdown of the efforts and their evolution during the optimization should give a full understanding of the improvements made.

Finally, the pressure coefficient $\left(C_{p}=\frac{p-p_{\infty}}{\frac{1}{2} \rho_{\infty} V_{\infty}}\right)$ is computed on the surface of the nacelle and plotted on Fig. 10 .

It appears that the low pressure coefficient area on the inner wall of the secondary nozzle has been reduced on the optimal shape. This is in good agreement with the velocity reduction that has been observed on the Mach fields. In 


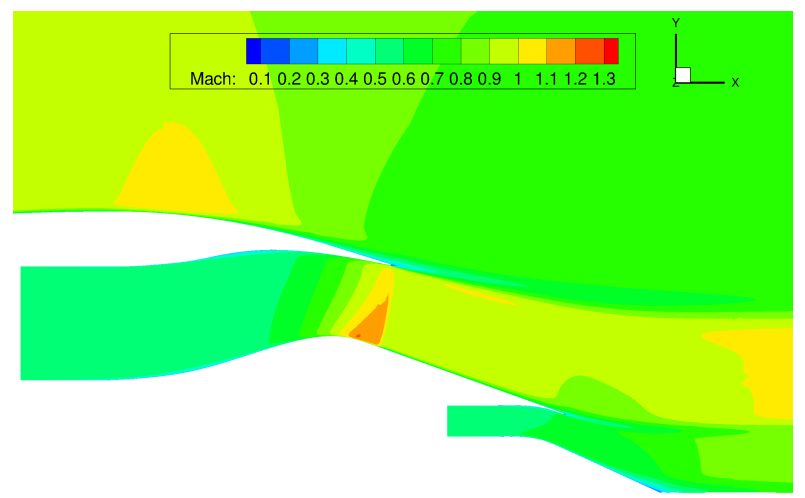

(a)

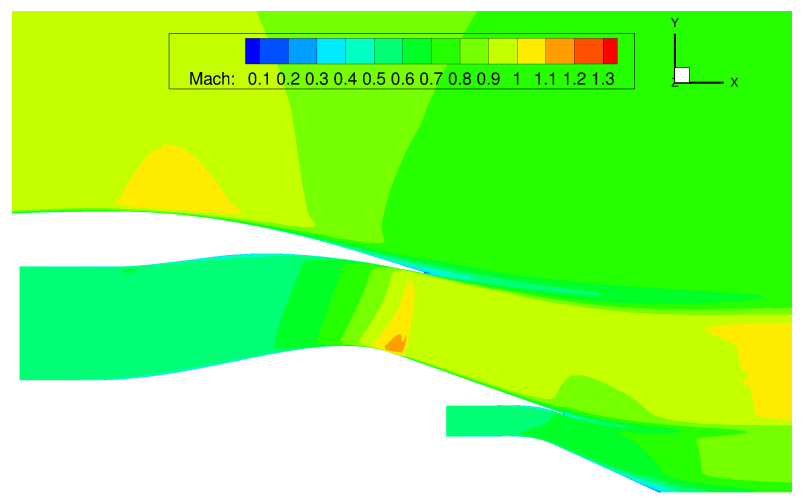

(b)

Fig. 9 Mach field in a slice $z=0$ of the reference (a) and optimized (b) nozzle shapes

conclusion, the surface mapping of $C_{p}$ shows how shape modifications can alter skin pressure, and gives complementary informations on the nozzle configurations to the designer.

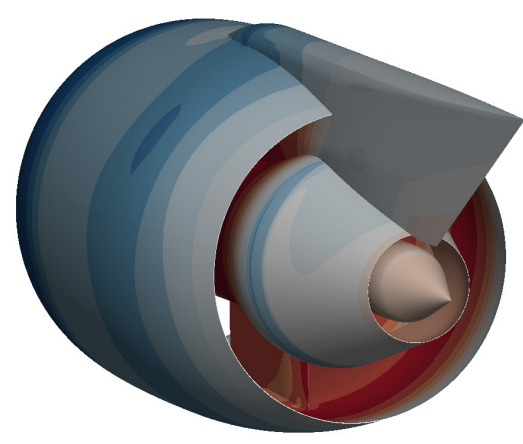

(a)
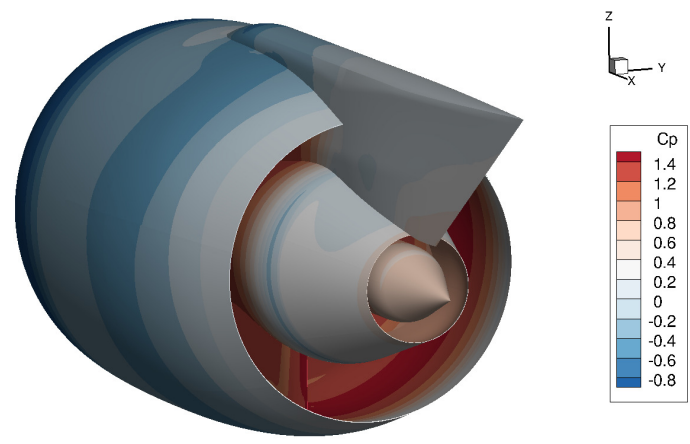

(b)

Fig. 10 Skin pressure on the reference (a) and optimized (b) nozzle shapes

\section{Conclusions and perspectives}

This paper presents aerodynamic shape optimizations based on industrial design tools and applied to aircraft engine nozzles. First, a CAD model of industrial complexity representing the IPPS (engine nacelle, pylon and bifurcations) is presented. Integrating such design tool in optimization workflows is challenging, that is why a dedicated approach is presented. This approach allows to reduce the dimensionality of the problem and enables the use of gradient-free algorithms or the computation of finite-difference sensitivities. Then, this "convex combination" method is assessed on an analytical case and an inverse optimization problem. This simple application shows that the dimensionality reduction meets the a priori expectations and that the method is promising for an application on more complex cases. Consequently, a workflow based on industrial design tools comprising CAD, meshing and aerodynamic computations is implemented. This process aims at improving the efficiency of a dual-separate-flow turbofan nozzles. When submitted to an optimizer, this first case of industrial complexity validates the convex combination approach and the workflow. Interesting efficiency improvements are achieved, although the combination database contains simple shapes. In addition, useful informations are returned to the designer thanks to the combination coefficients.

The present work has several perspectives. First, the combination method has been applied with a database of 3 CAD shapes only. The number of shapes in the database can be increased, or more importantly, they can be exchanged or completed, based on previous optimization results. In fact, this method introduces an original iterative design process, based on fast optimizations and learning, in interaction with the designer. 
Then, the use of gradient-based descent algorithm and finite-differences can be discussed. The dimensionality reduction associated to convex combination enables to consider various optimization approaches. This remains true, even if the database has more expert configurations than presented here. Therefore, Surrogate-Based Optimization (SBO) or Genetic Algorithms (GA) will be considered in future works.

Besides, it appears that the main problem, the integration of a complex CAD model in an optimization workflow, has been sucessfully solved. By reducing the dimensionality of the problem, non-differentiated tool used in industrial design processes can now be integrated in the optimization workflow as well. Thus, it becomes possible to enhance the fidelity of the physics involved in the optimization. For instance, aerodynamic computations can be replaced by coupled aeropropulsive simulations. This would give a direct access to the fuel consumption of the engine, and enable its definition as objective function.

\section{Acknowledgements}

The studies presented in this article have used the ONERA-Airbus-SAFRAN elsA software whose development is partially funded by Airbus, Safran and ONERA, which are co-owners of this software.

\section{References}

[1] Mohammadi, B., and Pironneau, O., Applied shape optimization for fluids, Oxford university press, 2010.

[2] Carrier, G., Destarac, D., Dumont, A., Méheut, M., Salah El Din, I., Peter, J., Khelil, S., Brezillon, J., and Pestana, M., "Gradient-Based Aerodynamic Optimization with the elsA Software," 2014.

[3] Méheut, M., Destarac, D., Khelil, S. B., Carrier, G., Dumont, A., and Peter, J., "Gradient-Based Single and Multi-points Aerodynamic Optimizations with the elsA Software," 53rd AIAA Aerospace Sciences Meeting, 2015.

[4] Meheut, M., Dumont, A., Carrier, G., and Peter, J., "Gradient-Based Optimization of CRM Wing-alone and Wing-body-tail Configurations by RANS Adjoint Technique," 54th AIAA Aerospace Sciences Meeting, 2016.

[5] Dumont, A., Méheut, M., Baumgärtner, D., and Bletzinger, K.-U., “Aerodynamic Shape Optimization Progress on ADODG Benchmark Problems Using the elsA Software," 35th AIAA Applied Aerodynamics Conference, 2017.

[6] Merle, A., Stueck, A., and Rempke, A., "An Adjoint-based Aerodynamic Shape Optimization Strategy for Trimmed Aircraft with Active Engines," 35th AIAA Applied Aerodynamics Conference, 2017.

[7] Bell, R. A., and Cedar, R. D., "An inverse method for the aerodynamic design of three-dimensional aircraft engine nacelles," Third International Conference on Inverse Design Concepts and Optimization in Engineering Sciences (ICIDES-3), 1991, pp. 405-417.

[8] Lambert, P.-A., "Optimisation de formes en aérodynamique: application à la conception des nacelles de moteurs civils," Ph.D. thesis, Ecole Centrale Paris, 1995.

[9] Toubin, H., Salah El Din, I., and Meheut, M., "Multipoint aerodynamic high fidelity shape optimization of an isolated engine nacelle," 52nd AIAA Aerospace Sciences Meeting, AIAA SciTech, 2014.

[10] Goulos, I., Stankowski, T., MacManus, D., Woodrow, P., and Sheaf, C., "Civil turbofan engine exhaust aerodynamics: Impact of bypass nozzle after-body design,” Aerospace Science and Technology, Vol. 73, 2018, pp. 85 - 95.

[11] Gray, J. S., Mader, C. A., Kenway, G. K., and Martins, J. R., "Modeling Boundary Layer Ingestion Using a Coupled Aeropropulsive Analysis," Journal of Aircraft, 2017, pp. 1-9.

[12] Gray, J. S., Kenway, G. K., Mader, C. A., and Martins, J., "Aero-propulsive Design Optimization of a Turboelectric Boundary Layer Ingestion Propulsion System,” 2018 Aviation Technology, Integration, and Operations Conference, ????

[13] Dassault Systèmes, "CATIA V5,", 1998. URLhttps://wWw.3ds.com/products-services/catia/

[14] Bagy, S., Mohammadi, B., Méheut, M., Lallia, M., and Coat, P., “Towards CAD-based shape optimization of aircraft engine nozzles," EUROGEN Conference, 2019.

[15] Candel, S., Mécanique des fluides, Dunod, 1990.

[16] Wiart, L., Atinault, O., Grenon, R., Paluch, B., and Hue, D., "Development of NOVA aircraft configurations for large engine integration studies," 33rd AIAA Applied Aerodynamics Conference, 2015, p. 2254. 
[17] Empresarios Agrupados, "PROOSIS,", 2012. URL https://www.ecosimpro.com/products/proosis/

[18] Adams, B., Bohnhoff, W., Dalbey, K., Eddy, J., Eldred, M., Gay, D., Haskell, K., Hough, P., and Swiler, L., "DAKOTA, a multilevel parallel object-oriented framework for design optimization, parameter estimation, uncertainty quantification, and sensitivity analysis: version 5.0 user's manual," Sandia National Laboratories, Tech. Rep. SAND2010-2183, 2009.

[19] Vanderplaats Research and Development, "DOT Users Manual, Version 4.20,” 1995.

[20] ANSYS, "ICEM,", 2015. URLhttps://wWw. ansys.com

[21] Cambier, L., Heib, S., and Plot, S., “The Onera elsA CFD software: input from research and feedback from industry," Mechanics \& Industry, Vol. 14, No. 3, 2013, pp. 159-174.

[22] Benoit, C., Peron, S., and Landier, S., "Cassiopee: a CFD pre- and post-processing tool," Aerospace Science and Technology, Vol. 45, 2015, pp. 272-283. 